\title{
Self-Criticism, Neediness, and Distress Among Women Undergoing Treatment for Breast Cancer: A Preliminary Test of the Moderating Role of Adjustment to Illness
}

\author{
Rui C. Campos
}

University of Évora

Avi Besser

Sapir Academic College

Raquel Ferreira

University of Évora

Sidney J. Blatt

Yale University

$\bar{A}$ diagnosis of cancer can be extremely stressful, and for that reason, cancer patients' adjustment has been widely studied. Little is known, however, about how patients' personality vulnerabilities affect their adjustment to cancer. The present study examined the moderating role of several psychological strategies of adjustment to cancer in the associations between the personality predispositions of self-criticism and neediness and distress among women diagnosed with breast cancer. Portuguese women who had been diagnosed with breast cancer for the first time $(\mathrm{n}=50)$ completed the Depressive Experiences Questionnaire, the Hospital Anxiety and Depression Scale, and the Mini-Mental Adjustment to

Rui C. Campos and Raquel Ferreira, Department of Psychology, University of Évora, Portugal; Avi Besser, Department of Behavioral Sciences and the Center for Research in Personality, Life Transitions, and Stressful Life Events, Sapir Academic College, D. N. Hof Ashkelon, Israel; Sidney J. Blatt, Departments of Psychiatry and Psychology, Yale University.

We thank all of the participants in this study. Grateful thanks are also extended to the anonymous reviewers for their constructive suggestions and comments on a draft of this article. This article is based in part on Raquel Ferreira's Master's thesis, which was completed under the supervision of Rui C. Campos.

Correspondence concerning this article should be addressed to Rui C. Campos, Department of Psychology, University of Évora, Apartado 94, Évora, Portugal 7002-554. E-mail: rcampos@uevora.pt or to Avi Besser, Department of Behavioral Sciences and Center for Research in Personality, Life Transitions, and Stressful Life Events, Sapir Academic College, D. N. Hof Ashkelon 79165, Israel. E-mail: besser@mail.sapir.ac.il 
Cancer Scale questionnaires. Both self-criticism and neediness were found to be associated with increased levels of distress, with a stronger association observed for neediness. Hierarchical regressions indicated that more adaptive adjustment to cancer (i.e., low levels of helplessness/hopelessness, low levels of anxious preoccupation, high levels of fatalism, and high levels of fighting spirit) moderates the association between neediness and distress. There was no evidence that any of the adjustment variables had any mediating effect on the relationship between the personality variables (self-criticism and neediness) and distress. Results are discussed in the context of personality vulnerability and maladaptive psychological response to the disease as a stressful life event. Implications for treatment are discussed. Though promising, the results are preliminary and more research on larger samples is warranted.

Keywords: breast cancer, neediness, self-criticism, adjustment to illness, distress

Cancer is a life-threatening disease with powerful psychological implications. Breast cancer patients are at risk for affective disorders, such as anxiety and depression (Bleiker, Pouwer, Ploeg, Leer, \& Ader, 2000). The psychological vulnerability associated with cancer is significant; evidence suggests that between $15 \%$ and $40 \%$ of cancer patients experience clinically significant levels of anxiety, depression, or both (e.g., Parle, Jones, \& Maguire, 1996). In addition, between $20 \%$ and $30 \%$ of cancer patients remain in a distressed state long after the initial diagnosis. In Portugal, breast cancer is the primary cause of death among women ages 35-54 (Portuguese General Directorate of Health, 2007). Over the last decade, there has been growing interest in resiliency and the capacity for coping with stress and the stress of cancer in particular (e.g., review by Johansson, Rydén, \& Finizia, 2011). Basic personality predispositions seem to play a significant role in the level of adaptive coping with stress, especially in the context of an individual's response to the diagnosis of cancer.

The aim of the present study is to investigate the moderating roles of several psychological adjustment strategies in the context of cancer by examining the associations between the two basic personality predispositions defined in Blatt's personality theory (self-criticism and neediness; for a review, see Blatt, 2008) and distress among women recently diagnosed with breast cancer. The present study focuses on two central personality constructs that have not been studied previously in relation to the psychological adjustment and distress of cancer patients.

\section{ADAPTATION TO CANCER AND DISTRESS}

The conceptualization of coping presented by Lazarus and Folkman (1984) defines coping as the cognitive and behavioral effort exerted to manage specific external or internal demands. Consistent with this theory of 